\title{
O ACORDO DE PARIS: UMA ANÁLISE DESCRITIVA
}

Deborah Martins de Carvalho - deborah_martins08@ hotmail.com Universidade Federal de Minas Gerais

Aline Carolina Cirilo Coelho - alinescout@ gmail.com

Universidade Federal de Minas Gerais

Raiane Soares da Silva Martins - raianessm@gmail.com

Universidade Federal de Minas Gerais

Karoline Rodrigues Costa - karolrodriguescosta@gmail.com

Universidade Federal de Minas Gerais 


\section{RESUMO}

O objetivo deste trabalho é realizar uma avaliação crítica do Acordo de Paris. Inicialmente foi descrita a evolução do Protocolo de Quioto através da análise de documentos oficiais da Convenção-Quadro das Nações Unidas sobre Mudanças Climáticas (UNFCCC), além de artigos acadêmicos e matérias jornalísticas. Por se tratar do primeiro tratado que discutiu e propôs metas de redução de emissão de gases de efeito estufa, foi feito um paralelo com o quê espera-se do acordo seguinte. Com base nas questões levantadas na análise desse protocolo, foram discutidas as expectativas para o período pós-Quioto e para o novo acordo proposto em novembro de 2015.

\section{INTRODUÇÃO}

A preocupação com as mudanças climáticas globais é uma realidade para o mundo desde 1988 quando da criação do Painel Intergovernamental para Mudanças Climáticas (IPCC), num esforço conjunto da UNEP (Programa das Nações Unidas para o Meio Ambiente) e da WMO (Organização Meteorológica Mundial). Mas foi em 1992 quando, na Conferência das Nações Unidas sobre o Meio Ambiente e o Desenvolvimento, ocorrida na cidade do Rio de Janeiro, foi acordado a Convenção-Quadro das Nações Unidas sobre a Mudança do Clima, também conhecida como UNFCCC, que tal problema ganhou reconhecimento ainda maior.

Este tratado foi firmado por 197 Partes e tem como objetivo a estabilização das emissões de gases causadores do efeito estufa(GEE) de forma que os mesmos não causem interferência ao meio ambiente (UNFCCC, 2016a). Através do tratado foi criado o Protocolo de Quioto em 1997, que veio a ser o principal acordo firmado pelas Partes até então.

O Protocolo de Quioto teve como finalidade o estabelecimento de metas de redução de emissões dos GEE aos países-membros. No entanto, tais metas foram diferenciadas com relação ao nível de desenvolvimento dos países, deixando recair sobre cada um deles uma responsabilidade diferente na solução do problema. Levando em consideração a responsabilidade histórica das emissões dos países desenvolvidos (contidos no Anexo I), devido à maior contribuição dos mesmos para com as emissões, 


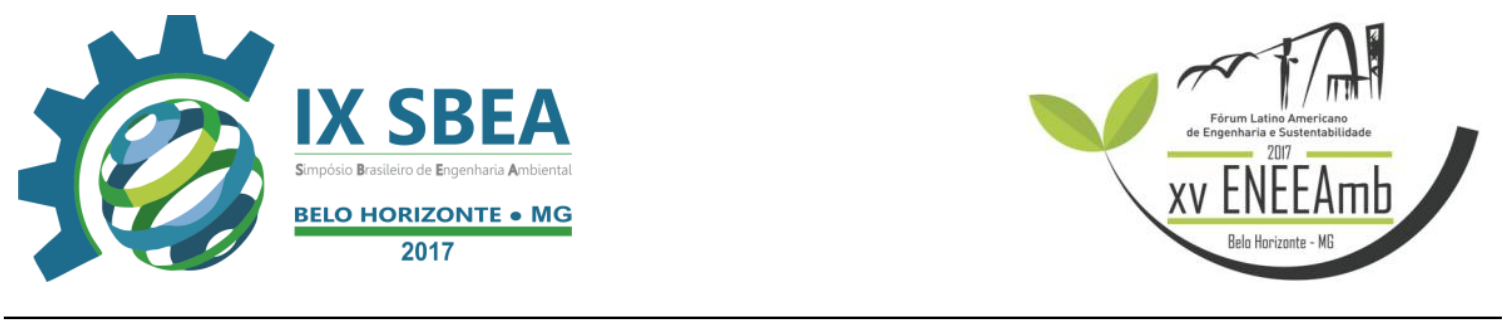

as metas foram destinadas a esses países. O Protocolo de Quioto entrou em vigor em 2005 adotando as regras estabelecidas na sétima Conferência das Partes (COP-7) em Marraquexe em 2001 tendo como primeiro período de compromisso, os anos entre 2008 e 2012 (UNFCCC, 2016 b).

A fim de que os países cumprissem seus objetivos, foram criados mecanismos no primeiro momento do protocolo, que permaneceram no segundo período, sendo eles: o Comércio Internacional de Emissões (CIE), o Mecanismo de Desenvolvimento Limpo (MDL) e a Implementação Conjunta (IC).

Os mecanismos ajudam as partes não só a cumprir suas metas como também têm um caráter rentável. O CIE permite aos países que possuem unidades de emissões além da quantidade que lhes é atribuída, a venda desse excesso àqueles que estão abaixo de seu objetivo de redução de emissão. Cria-se então uma nova mercadoria, o Carbono. Sendo esse o principal gás emitido, os outros gases têm o seu valor convertido ao valor equivalente de carbono.

As transações realizadas com as vendas de carbono equivalente deram origem ao Mercado de Carbono. De acordo com Johannsdottir e McInerney (2016), tal mercado além de maximizar os esforços para reduzir as emissões de GEE, estimulam investimentos em tecnologias inovadoras de baixo consumo de carbono e de energia.

Até mesmo os países que não têm metas estabelecias possuem uma maneira de cooperar com os países desenvolvidos. Através do MDL, que é uma contribuição voluntária significativa dos países em desenvolvimento, leva-se em consideração o princípio do Protocolo de Quioto das responsabilidades comuns, mas diferenciadas (Frondizi, 2009). Neste caso, são desenvolvidos de projetos e tem como responsável a iniciativa do empresariado. As atividades de projeto de MDL nos países em desenvolvimento têm que apresentar benefícios reais, mensuráveis e de longo prazo e estar diretamente relacionadas aos gases de efeito estufa, podendo reduzir as emissões de GEE ou aumentar a remoção de $\mathrm{CO}_{2}$.

O terceiro mecanismo de flexibilização, a Implementação Conjunta, dá a oportunidade a um país desenvolvido de poder implementar projetos que levem à redução de emissões em um outro país, também desenvolvido, no qual os custos com a redução 
da poluição são menores. A IC é uma maneira das Partes para cumprir uma parte dos seus compromissos de Quioto através de um meio flexível e eficaz em termos de custos, enquanto a Parte anfitriã se beneficia do investimento estrangeiro e da transferência de tecnologia (Lucas et al., 2011).

Um segundo período de compromisso foi acordado em 2012 em Doha, no Qatar, onde as Partes assumiram novas metas que começaram em 2013 e têm previsão de término para 2020. As metas de redução de emissões que antes assumiam uma média de 5\% com relação às emissões de 1990 foram aumentadas para uma média de $18 \%$ até o ano final de período. Além disso a composição dos países não permaneceu a mesma do primeiro grupo de países, neste caso, alguns países como Japão e Canadá não possuem mais compromisso de redução.

Ao final desse período iniciar-se-á uma nova etapa na luta contra as mudanças climáticas marcado pelo Acordo de Paris. Tal acordo foi negociado em dezembro de 2015 e visa reduções de emissões a partir de 2020. Pela primeira vez todos os países, independentemente da sua responsabilidade histórica, propõem-se a assumirem metas de redução visando uma causa comum. O objetivo central é limitar o aumento da temperatura global a $1,5^{\circ} \mathrm{C}$ até o final do século XXI.

Tendo em vista os acontecimentos já ocorridos e as novas perspectivas para o mundo em relação aos acordos climáticos elaborou-se o presente artigo para avaliar o desempenho do Protocolo de Quioto até então e em sua terceira seção são feitos prognósticos para o Acordo de Paris considerando-se o comportamento histórico mundial. Por fim, são sistematizadas algumas considerações, modo a concluir sobre o tema.

\section{METODOLOGIA}

Foram analisados diversos documentos oficiais da Convenção-Quadro das Nações Unidas sobre Mudanças Climáticas (UNFCCC), além de artigos acadêmicos e matérias jornalísticas de meios confiáveis que envolvessem tanto o posicionamento de autoridades no assunto e dos países quanto às mudanças climáticas, como as consequências econômicas e ambientais deste posicionamento. 


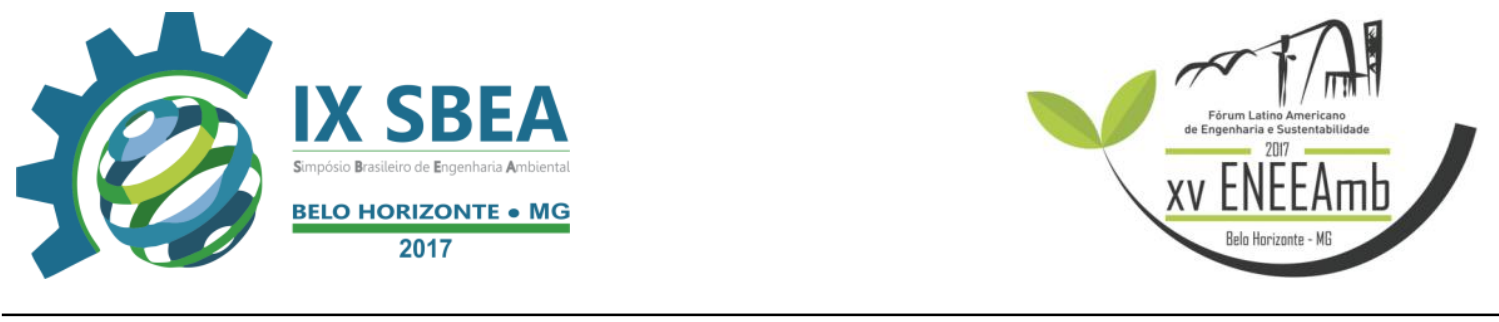

Através do estudo dessas informações, foi possível discutir e avaliar o desemprenho do protocolo de Quioto e levantar expectativas de como será a performance do tratado posterior, vigente no mundo o Acordo de Paris.

\section{RESULTADOS E DISCUSSÃO}

\subsection{O desempenho do Protocolo de Quioto}

O Protocolo de Quioto visa, do ponto de vista econômico, a eficiência. Sendo assim tal eficiência é garantida através dos mecanismos de flexibilização estabelecidos em sua composição. A cota de redução de emissão aplica-se somente para os países desenvolvidos, cotas que por sua vez, podem ser negociadas pelos países entre si. Em consequência, os países nos quais o custo de redução de emissão é muito elevado podem comprar cotas de emissão daqueles países onde o custo de redução é relativamente menor. Adicionalmente, o Protocolo de Quioto permite que os países que não conseguirem atingir a sua meta comprem Certificados de Emissão Reduzida (CER) de países em desenvolvimento, cujas emissões podem ser reduzidas a custos ainda menores.

Neste contexto, haverá países que reduzirão suas emissões além da cota estabelecida, devido a seus baixíssimos custos de redução, e países que extrapolarão a mesma, devido a seus custos relativamente mais elevados. Contudo, espera-se que, no total, a cota mundial seja atingida, tendo em vista quo problema é em escala global, garantindo a eficiência do tratado (Lucas et al. 2016).

Do ponto de vista ambiental a sua eficiência é questionável, uma vez que as mudanças climáticas que ocorrerão pela interferência antrópica causarão mais danos e prejuízos aos países em desenvolvimento. Tal grupo é constituído por países que não possuem uma economia consolidada que suporte tantas mudanças e não possuem recurso para uma possível intervenção. Pode-se ilustrar tal realidade com a situação vivida pela população de Kiribati, que sofre com o aumento do nível do mar e sofre risco de perder grande parte de sua área para o oceano (BBC, 2015).

Além disso, outro fato alvo de muitas discussões é a compensação de emissões por meio de replantio de árvores. A potencial reversibilidade do ciclo do carbono, 


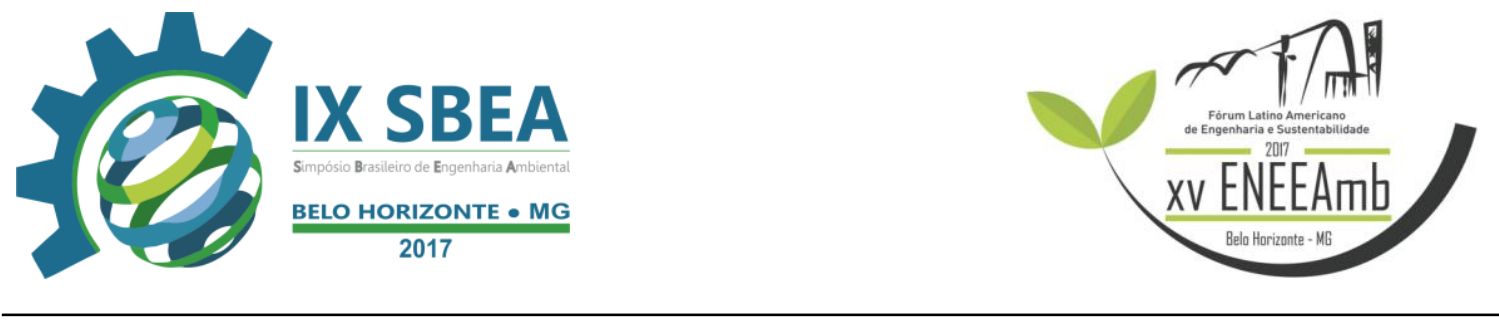

conhecida como "problema da permanência" que, segundo Maréchal e Hecq (2005), transforma atividades de florestamento em possível recurso de emissão em rede, quando no caso de incêndios, cortes ilegais, que contribuem para o aumento de $\mathrm{CO}_{2}$ na atmosfera.

Um outro problema que atrai numerosas críticas é o fato de que a ratificação do protocolo foi feita por algumas nações e outras não. Estas alegavam uma possível perda para a economia, como foi o caso dos Estados Unidos da América até 2014. Agindo desta forma, contribuía para a perda de eficiência do documento, uma vez que tal país é o principal emissor mundial; de modo semelhando os países em desenvolvimento podem, adicionalmente, aumentar suas emissões devido à fuga de países desenvolvidos.

De acordo com Diniz (2007) existe a chance de os investidores favorecerem os países em desenvolvimento devido a uma legislação ambiental menos rigorosa e à ausência de compromissos ambientais. A ausência de um mecanismo de prevenção de fugas sob a forma de sanções e restrições comerciais entre países é um problema potencial num protocolo se não houver uma quase total adesão.

A análise de alguns dos problemas e questionamentos citados acima pode ajudar a resolver as ineficiências geradas pelo protocolo. Desta maneira pode-se estudar a eficiência do Acordo de Paris do ponto de vista econômico para o problema do Aquecimento Global sem, todavia, deixar de lado a eficiência ambiental.

\subsection{Expectativas para o acordo de Paris}

Levando em consideração os pontos positivos e negativos, os questionamentos e metas do Protocolo de Quioto intenta-se verificar a possível eficiência do Acordo de Paris. Inicialmente é possível perceber a maior adesão das Partes em associar ao acordo em relação anterior.

O Protocolo de Quioto entrou em vigor em 2005, 8 anos após ser aberto para assinaturas, pois era necessário que a soma das poluições de cada país participante contabilizasse $55 \%$ do total.

No caso do Acordo de Paris, firmado em 2015, menos de um ano depois, atingiuse o limite mínimo de adesões para entrar em vigor, depois que 72 países ratificaram o texto, responsáveis por 56,72\% das emissões de GEE (Girardi, 2016). De acordo com 
Espinosa (2016), a precoce resposta ao acordo é um sinal político de que todas as nações do mundo estão dedicadas a uma ação global decisiva sobre as mudanças climáticas.

Além do aspecto da velocidade, o acordo também contou com assinaturas inéditas que fazem diferença no montante de emissões dos GEE, à medida que neste momento existe a participação dos países em desenvolvimento. Um exemplo é a participação da China que tem a intenção de, até 2030, reduzir cerca de 65\% dos GEE com relação aos níveis de 2005 (UNFCCC, 2015). Desta forma, o aumento das emissões por países em desenvolvimento é assim controlado.

O Acordo de Paris, assim como o Protocolo de Quioto, representa um desafio econômico único. Tendo em vista a participação global, os mecanismos de flexibilização se tornam ferramentas ainda mais relevantes para que as metas sejam alcançadas. $\mathrm{O}$ Mercado de Carbono tende a ter importância efetiva no desenvolvimento do futuro climático.

Todos os países que participam do acordo apresentaram à UNFCCC a pretendida contribuição nacionalmente determinada (INDC, na sigla em inglês), sendo que para serem atingidas exigirão ambiciosas reduções de emissão de GEE. Segundo Johannsdottir e McInerney (2016), os países serão forçados a apresentar políticas para cumprir estas metas, e os esquemas nacionais de determinação do preço do carbono e o desenvolvimento futuro dos Mercados de Carbono poderão surgir como resposta.

O Acordo de Paris afeta diretamente o esquema de produção do setor industrial dos países. Isso porque, para atingir as metas de redução, os investimentos financeiros devem estar voltados para as tecnologias de baixo carbono. Para atrair capital a tais investimentos, os subsídios aos combustíveis fosseis precisam ser reduzidos, além da necessidade de implementação de políticas que levem as empresas a aumentarem a transparência e a divulgação e refletirem os riscos operacionais, físicos e de litígios decorrentes da exposição ao carbono em seus resultados financeiros (McInerney et al., 2016).

Em relação aos aspectos ambienteis relacionadas ao Acordo de Paris, as expectativas são grandes. A meta de assegurar o aumento da temperatura média global em $2{ }^{\circ} \mathrm{C}$ abaixo dos níveis pré-industriais e prosseguir os esforços para limitar o aumento 
da temperatura a até $1,5{ }^{\circ} \mathrm{C}$ acima dos níveis pré-industriais faz com que a sejam reduzidos significativamente os riscos e impactos das alterações climáticas.

O IPCC, em apoio ao acordo, tem a intenção de redigir novos relatórios levado em consideração as metas citadas. O relatório estará disponível em 2018 segundo dados da UNFCCC. A intenção é estudar o comportamento do clima com o aumento controlado da temperatura e então prever o que pode acontecer com o ambiente global.

No entanto, de acordo com Watson et al. (2016), mesmo se todas as metas para 2030 forem cumpridas, não será suficiente para evitar o aquecimento acima de $2{ }^{\circ} \mathrm{C}$. É importante que os países não se contentem apenas com os compromissos assumidos no Acordo de Paris. Tal realidade é um problema ambiental significativo e é preciso dar atenção às consequências que podem ser acarretadas. Ainda assim, as perspectivas são melhores quando comparadas àquelas relacionadas ao Protocolo de Quioto.

\section{CONCLUSÕES}

Os problemas ambientais ligados ao aquecimento global são substanciais. Esta é a atual realidade com que o mundo precisa lidar. Tendo em vista a grandeza do problema, soluções drásticas devem ser tomadas. Com isso são criados os tratados visando uma meta em comum para todos os países.

Todos os países possuem seus interesses distintos uns dos outros e alinhar esses interesses de forma a atingir a uma meta em comum é um processo que demanda tempo. Entretanto este tempo não se faz presente no atual cenário. Desta forma, é necessário ainda mais a conscientização dos países e políticas voltadas para evitar a emissão de GEE e consequentemente o aquecimento global.

O presente artigo tentou analisar as expectativas que surgem ao Acordo de Paris no período pós-Quioto. A recente aprovação do acordo mostra que ainda não são encontradas tantas informações e estudos ainda estão sendo produzidos a fim de expor a real eficiência do mesmo. Ainda assim, pode-se afirmar que Acordo de Paris demanda medidas desafiadoras tanto no aspecto político quanto econômico aos países participantes. 


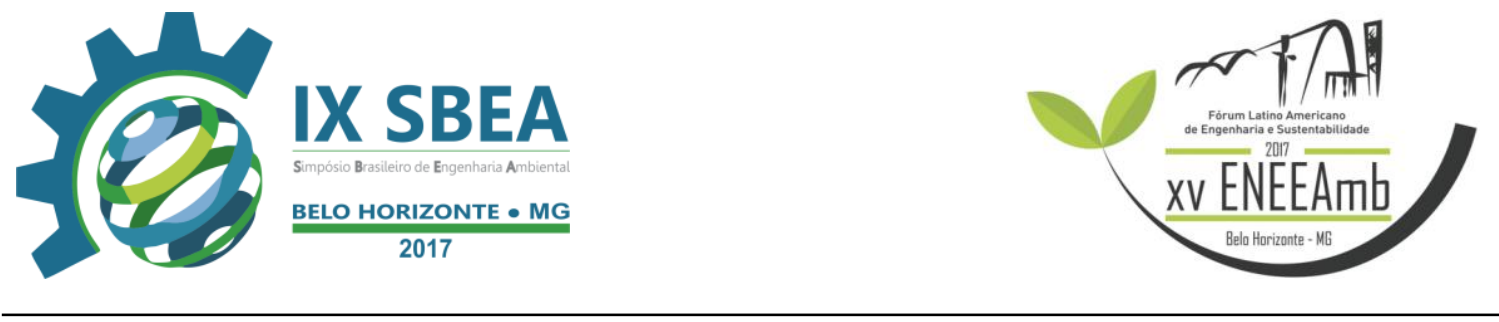

A rápida adesão de um número considerável de países indica que a consciência ambiental que começou a surgir anos atrás está, de fato, enraizada. O problema que antes levantava questões sobre sua veracidade, agora é indubitável. O Acordo de Paris pode não ser adequado para este desafio, porém é um passo dado a um futuro mais limpo, e pode ser melhorado nos debates e estudos que serão produzidos. O que não é prudente são nações de todo o mundo fecharem os olhos e continuarem com a poluição desenfreada.

\section{REFERÊNCIAS BIBLIOGRÁFICAS}

BBC. Kiribati's climate change Catch-22. 2015. Disponível em: <http://www.bbc.com/news/world-asia-35024046>. Acesso em: 08 dez. 2016

DINIZ, E. M. Lessons from the Kyoto protocol. Ambiente \& sociedade, v. 3, n. SE, p. 0-0, 2007.

ESPINOSA, P. Paris Enters into Force - Celebration and Reality Check. 4 nov. 2016.

Disponível em: <http://newsroom.unfccc.int/paris-agreement/paris-agreement-entersinto-force-celebration-and-reality-check/>. Acesso em: $10 \mathrm{dez} .16$

FRONDIZI, I. M. R. L. (Coord.). O mecanismo de desenvolvimento limpo: guia de orientação. Rio de Janeiro: Imperial Novo Milênio: FIDES, 2009.

GIRARDI, G. Acordo de Paris atinge adesão mínima necessária e entra em vigor em 30 dias. Estadão. São Paulo. 05 out. 2016. Disponível em: < http://sustentabilidade.estadao.com.br/blogs/ambiente-se/acordo-de-paris-atinge-adesaominima-e-entra-em-vigor-em-30-dias/>. Acesso em: $10 \mathrm{dez} .16$

JOHANNSDOTTIR, L.; MCINERNEY, C. Calls for Carbon Markets at COP21: a conference report. Journal of Cleaner Production, v. 124, p. 405-407, 2016.

LUCAS, N. D., SALES, S. A. M. Evidências do Protocolo de Quioto no Brasil: Uma análise exploratória descritiva. Revibec: revista de la Red Iberoamericana de Economia Ecológica, v. 16, p. 0033-48, 2011.

MARÉCHAL, K.; HECQ, W. Temporary credits: A solution to the potential nonpermanence of carbon sequestration in forests?. Ecological Economics, v. 58, n. 4, p. 699-716, 2006. 
MCINERNEY, C.; JOHANNSDOTTIR, L. Lima Paris Action Agenda: Focus on Private Finance-note from COP21. Journal of Cleaner Production, v. 126, p. 707-710, 2016. UNFCCC, 2015. Enhanced actions on climate change: China's intended nationally determined contributions. Disponível em: <http://www4.unfccc.int/Submissions/INDC/Published\%20Documents/China/1/China's\%20INDC\%20\%20on\% 2030\%20June\%202015.pdf>. Acesso em: 10 dez. 16

UNFCCC, 2016a. About UNFCCC. Disponível em:
<http://newsroom.unfccc.int/about/>. Acesso em: 24 nov. 16.

UNFCCC 2016b. Kyoto Protocol. Disponível em: <http://unfccc.int/kyoto_protocol/items/2830.php>. Acesso em: 24 nov. 16 WATSON, R., CARRARO, C., CANZIANI, P., NAKICENOVIC, N., MCCARTHY, J. J., GOLDEMBERG, J., \& HISAS, L. The Truth About Climate Change. 2016. 